\title{
Thrombocytopenia in Experimental Trypanosomiasis
}

\author{
Charles E. Davis, Robert S. Robbins, Ruchard D. Welleer, and \\ Abraham I. Braude \\ From the Departments of Pathology and Medicine, University of California, \\ San Diego 92103
}

A B S T R A C T The effect of experimental trypanosomiasis on coagulation was studied because a patient in this hospital with Rhodesian trypanosomiasis developed thrombocytopenia with disseminated intravascular coagulation. Rats injected intraperitoneally with this strain of Trypanosoma rhodesiense consistently developed trypanosomiasis and severe thrombocytopenia without changes in hematocrit or concentration of fibrinogen or fibrin split products. At the time of $50 \%$ mortality (4-5 days) mean platelet counts per cubic millimeter of infected rats were $18,000 \pm 9,000( \pm 2 \mathrm{SEM}$ ) compared to $1,091,000 \pm 128,000$ in uninfected controls.

In vitro, concentrated trypanosomes and trypanosomefree supernates of disrupted organisms added to normal rat, rabbit, or human blood produced platelet aggregation within $30 \mathrm{~min}$. This platelet aggregation was not blocked by inhibitors of ADP, kinins, or early or late components of complement. In vivo thrombocytopenia also occurred in infected rabbits congenitally deficient in C6 and in infected, splenectomized rats.

Although the aggregating substance obtained from disrupted trypanosomes is heat-labile, it is active in the presence of complement inhibitors, suggesting that this trypanosomal product may be a protein enzyme or toxin. Since the phenomenon is independent of immune complexes, complement, ADP, and kinins, it appears to represent a new mechanism of microbial injury of platelets and the induction of thrombocytopenia.

\section{INTRODUCTION}

We studied the effect of experimental trypanosomiasis on coagulation factors because a patient in this hospital with Rhodesian trypanosomiasis almost died of disseminated intravascular coagulation $(\text { DIC })^{1}$ (1). Various

An abstract of a portion of this work appeared in Clin. Res. 1973. 21: 269.

Received for publication 16 July 1973 and in revised form 2 November 1973.

${ }^{1}$ Abbreviations used in this paper: DIC, disseminated severe infections may cause DIC, but it is usually associated with meningococcemia or septicemia due to gram-negative bacilli. Among the protozoan infections only severe falciparum malaria is well documented as a cause of this syndrome $(2,3)$.

We were surprised to find that $100 \%$ of rats infected with this Rhodesian strain developed severe thrombocytopenia without evidence of hemolysis or DIC. This dissociation of thrombocytopenia from DIC strongly indicates a direct toxic effect of the trypanosomes on platelets. Although it has been speculated that Plasmodium vivax may produce circulating toxins directly harmful to platelets (4), most studies suggest that the thrombocytopenia associated with malaria is due to DIC $(2,3)$, immunologic injury (5), or splenic pooling (6). In bacterial septicemias the thrombocytopenia is clearly associated with DIC (7).

In this study we reproduced platelet injury in vitro by whole trypanosomes and with soluble extracts of disrupted trypanosomes. Since the process is independent of immune complexes and complement and is not blocked by inhibitors of ADP or kinins, it appears to represent a new mechanism of microbial injury of platelets and thrombocytopenia.

\section{METHODS}

Experimental trypanosomiasis. Male 200-g Holtzman rats or $2.5-3.0 \mathrm{~kg}$ white New Zealand rabbits were injected i.p. with a $0.15 \mathrm{M} \mathrm{NaCl}$ dilution of infected, fresh rat blood containing $10-20,000$ trypanosomes, or more than 1,000 infective doses for rats. Syringe-passed trypanosomes maintained in rats were used in all experiments. During the first few passages this strain of $T$. rhodesiense became more virulent for rats, but the clinical syndrome and time of $\mathrm{LD}_{100}$ has been stable for $1 \mathrm{yr}$. Control animals received either an equal volume of normal rat blood or nothing. Normal rat blood caused no visible illness and no blood

intravascular coagulation; EM, electron microscopy; FSP, fibrin and fibrinogen split products; HCT, hematocrit; IBS, isotonic buffered saline; PRP, platelet-rich plasma; PSG, phosphate-buffered saline glucose solution. 
changes. Studies on splenectomized rats were performed after wounds were well healed and platelet counts had returned toward normal. Rabbits congenitally deficient in C6 were infected in the usual manner. Rats were exsanguinated by cardiac puncture at the time of study. Rabbits were bled from the central artery of the ear. Autopsies were performed on 18 rats at the time of $50 \%$ mortality. Serial sections of formaldehyde-fixed kidneys, livers, spleens, lung, hearts, and brains were stained with hematoxylin and eosin and by the periodic-acid Schiff method.

Hematological studies. Platelets and trypanosomes were counted by phase microscopy. Occasionally, nonmotile trypanosomes assume a spherical shape but can still be differentiated from platelets by careful observation. Microhematocrits (HCT) were determined by centrifugation of EDTAtreated blood in capillary tubes. Fibrinogen was measured by the method of Ratnoff and Menzie (8) but with citrated blood, and split products of fibrin and fibrinogen (FSP) were estimated by the staphylococcal clumping technique (9). The details of these procedures have been described in another publication (10).

In vitro platelet aggregation. Normal rat, rabbit or human blood was harvested in $0.008 \mathrm{mg} / \mathrm{ml}$ EDTA (EDTA : blood $=1: 9 ; \mathrm{vol}: \mathrm{vol}$ ). Blood from infected rats was harvested either in EDTA or $0.38 \mathrm{mg} / \mathrm{ml}$ sodium citrate $(1: 9 ; \mathrm{vol}: \mathrm{vol})$. Unless otherwise noted, EDTA and sodium citrate were always used at these standard anticoagulating concentrations. Trypanosomes were harvested from a visible layer on top of the buffy coat of infected rat blood and stored at $4^{\circ} \mathrm{C}$ until use. These concentrated trypanosomes were platelet-poor and contained no platelet aggregates.

To $1 \mathrm{ml}$ of normal blood harvested in EDTA, $0.1 \mathrm{ml}$ of concentrated trypanosomes $\left(10^{6}-10^{7} / \mathrm{mm}^{3}\right)$ or $0.1 \mathrm{ml}$ of normal, species-specific plasma was added. The tubes were rocked continuously at 36 oscillations/min on an Ames aliquot mixer. (Ames Co., Div. of Miles Lab, Inc., Elkhart, Ind.) at room temperature, and platelet counts and other determinations were performed at $30 \mathrm{~min}$. All platelets that could be counted accurately were incorporated in the tally, including platelets in small aggregates of four or five.

Investigation of the mechanism of platelet injury. The response of the platelet counts of infected animals to increasing trypanosomemia was measured by infecting 36 rats and exsanguinating at least 6 daily. Trypanosomes and platelets were counted by phase microscopy, and the results were plotted as platelet counts versus trypanosome counts. Six littermates were used as the normal controls. The "doseresponse" relationship of the aggregation phenomenon was measured by noting the effect of $0.1 \mathrm{ml}$ of serial 10 -fold dilutions of trypanosomes $\left(8.8 \times 10^{8} / \mathrm{mm}^{3}, 8.8 \times 10^{5} / \mathrm{mm}^{3}\right.$, and $8.8 \times 10^{4} / \mathrm{mm}^{3}$ ) on $1 \mathrm{ml}$ of a pool of normal rat blood. The negative control consisted of $0.1 \mathrm{ml}$ of normal plasma harvested from the same pool of rat blood.

Platelet aggregation was tested under several experimental situations designed to elicit the nature of the mediator of trypanosome-induced aggregation. It was likely that fairly large amounts of exogenous ADP from the trypanosomes and rat red cells were added to normal blood during the in vitro aggregation experiments. Accordingly, very large concentrations of ADP (up to $60 \mathrm{mg} / \mathrm{ml}$ ) were added to the EDTA-treated normal blood instead of trypanosomes. The ability of trypanosomes to induce platelet aggregation in whole blood or platelet-rich plasma (PRP) pretreated with $50 \mathrm{mM}$ adenosine was determined. Exoge- nous ADP was also removed by dialyzing the harvested trypanosomes overnight against phosphate-buffered saline and by separating the trypanosomes from rat blood in a QAE-50 Sephadex column with a phosphate-buffered salineglucose (PSG) solution (11). Dialyzed trypanosomes and column trypanosomes, which were completely free of rat erythrocytes and platelets, were washed with 10 vol of PSG, diluted to $8 \times 10^{8} / \mathrm{mm}^{3}$, and tested for the aggregating effect as described above.

In order to test whether complement components were required for trypanosome-induced platelet aggregation, the blood of rabbits congenitally deficient in $\mathrm{C} 6$ was drawn into $0.2 \mathrm{M}$ EDTA, a concentration sufficient to inactivate $\mathrm{C} 4$ and $\mathrm{C} 2$, and the usual concentration of trypanosomes was added.

Participation of kininlike substances in the platelet injury was checked by adding trypsin $(50-500 \mu \mathrm{g} / \mathrm{ml})$, alphachymotrypsin $(50-500 \mu \mathrm{g} / \mathrm{ml})$, or an equal volume of saline to harvested trypanosomes for $30 \mathrm{~min}$ at $37^{\circ} \mathrm{C}$. Lima bean trypsin inhibitor was then added to each sample of trypanosomes before addition of $0.1 \mathrm{ml}$ of the treated trypanosomes to $1.0 \mathrm{ml}$ of rat blood.

The effect of washing the trypanosomes before addition to normal blood was tested by washing the harvested trypanosomes from an aliquot of plasma three times in 10 vol of $0.15 \mathrm{M} \mathrm{NaCl}$. In the in vitro system, the washed trypanosomes, the trypanosome-free infected plasma concentrated five times, and the washings reduced in volume at $4^{\circ} \mathrm{C}$ in dialysis tubing to only twice the original plasma volume were tested for their ability to cause platelet aggregation.

$1 \mathrm{ml}$ of trypanosomes $\left(10^{\circ}\right)$ harvested from rat blood was sonicated in ice by means of a biosonic probe with one or two 10 -s bursts at $30 \%$ intensity. This short period disrupted approximately $90-95 \%$ of the trypanosomes. The sonicated material was then made trypanosome-free by centrifugation at $3,500 \mathrm{~g}$ for $20 \mathrm{~min} .1 \mathrm{ml}$ each of the untreated supernate, supernate preheated at $56^{\circ} \mathrm{C}$ for $30 \mathrm{~min}$, and supernate treated with trypsin or alpha-chymotrypsin was added to $1 \mathrm{ml}$ of normal rat blood.

Electron microscopy $(E M)$. Column-purified trypanosomes or trypanosome-free sonicate were added to tubes of citrated rat PRP and gently rocked at room temperature. Samples were withdrawn at $30 \mathrm{~min}$ and $90 \mathrm{~min}$ for fixation and preparation for EM, along with control samples of PRP.

For transmission EM the samples were added to equal volumes of $0.2 \%$ glutaraldehyde in isotonic buffered saline (IBS) centrifuged at $1,500 \mathrm{~g}$ for $15 \mathrm{~min}$ to form a loose pellet, fixed in $2 \%$ glutaraldehyde in IBS for $2 \mathrm{~h}$, postfixed in $2 \%$ osmium tetroxide in IBS for $1 \mathrm{~h}$, dehydrated, and embedded in Araldite (Ciba Products Co., Summit, N. J.). Sections were cut with a Porter-Blum MT-2B ultramicrotome and stained with lead citrate and uranyl acetate. They were examined with a Zeiss 9S electron microscope (Carl Zeiss, Inc., New York).

For scanning EM samples were pipetted onto glass cover slips and fixed in place with $0.2 \%$ glutaraldehyde in IBS for $15 \mathrm{~min}$, followed by $2 \%$ glutaraldehyde in IBS for 1 h. After dehydration in an ethanol-Freon series and processing in a Bomar SPC-900 critical point drying apparatus (The Bomar Co., Tacoma, Wash.), they were mounted on aluminum studs, coated with a 400-600- $\AA$ layer of gold-palladium, and examined with an Etec U-1 scanning electron microscope (Etec Corp., Hayward, Calif.). 


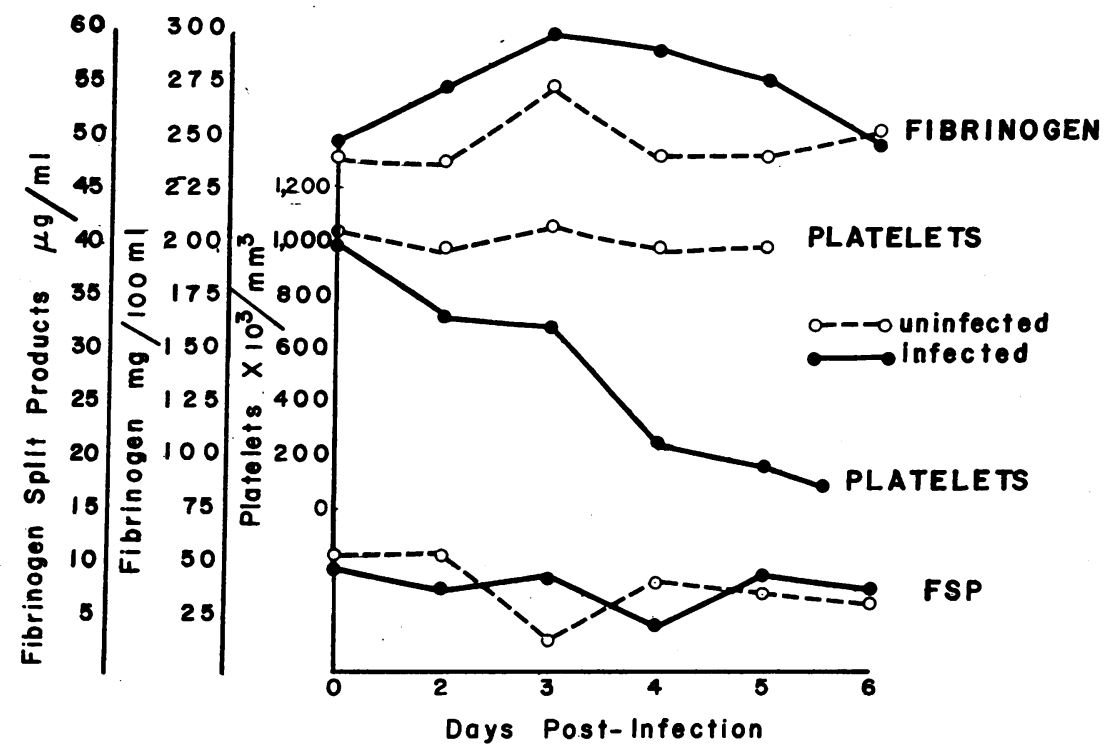

FIGURE 1 Effect of experimental trypanosomiasis on the platelet count, fibrinogen, and FSP concentration. Rats were infected with 10-20,000 trypanosomes intraperitoneally. Each day three normal and three infected rats were exsanguinated and studied until all rats were dead. Each point represents the mean platelet count and fibrinogen and FSP concentration of three rats.

\section{RESULTS}

\section{Experimental trypanosomiasis}

More than 500 rats and 50 rabbits were studied. All infected rats died between 96 and $144 \mathrm{~h}$ with no obvious signs of illness until shortly before death. Rabbits followed a more erratic course and died after a cachetic illness of 3-6 wk. The effect of experimental trypanosomiasis on the platelet count, Hст, and fibrinogen was determined $96 \mathrm{~h}$ after infection ( $24 \mathrm{~h}$ before the first spontaneous death occurred) and at $120 \mathrm{~h}$, the death point in rats not subjected to blood studies. As shown in a typical experiment in Table I, the Hcr and fibrinogen concentrations of infected rats did not differ from uninfected controls. The platelet counts, on the other hand, were markedly reduced in infected rats at $96 \mathrm{~h}$ and again fell sharply during the $24 \mathrm{~h}$ before death, when the number of trypanosomes in the blood was increasing rapidly. In fact an inverse relationship between the number of trypanosomes and platelets suggested a direct effect of trypanosomes or their products on the platelets. No platelet aggregates were ever noted in the platelet counts performed during the in vivo experiments.

Next a group of rats were studied daily to be certain that DIC with early consumption of fibrinogen and production of FSP was not responsible for the thrombocytopenia that occurred late in the disease. The range of FSP in more than 25 normal rats was $2-16 \mu \mathrm{g} / \mathrm{ml}$ with a mean of $8 \mu \mathrm{g} / \mathrm{ml} \pm 0.68(2 \mathrm{SEM})$. When three different rats were studied daily (Fig. 1) until all infected controls died, the only abnormality was a daily fall in platelets.

Gross and microscopic examinations of the kidneys, livers, spleens, lungs hearts, and brains of infected rats failed to demonstrate vascular lesions or fibrin deposition. Splenomegaly was the only finding. If the thrombocytopenia were due to removal of normal or injured platelets by the enlarged spleen, splenectomized animals

TABLE I

Effect of Experimental Trypanosomiasis on Platelet Count, Fibrinogen, and HCT

\begin{tabular}{lclc}
\hline & Platelet count & Fibrinogen & HCT \\
\hline 96 h after infection & $\times 10^{3} / \mathrm{mm}^{3}$ & $\mathrm{mg} / 100 \mathrm{ml}$ & $\%$ \\
$\quad$ & $335 \pm 114^{*}$ & $210 \pm 18$ & $41 \pm 3$ \\
$\quad$ Normal & $1,064 \pm 106$ & $185 \pm 9$ & $41 \pm 0.7$ \\
120 h after infection & $18 \pm 9$ & $257 \pm 40$ & $37 \pm 3.6$ \\
$\quad$ Infected & $1,091 \pm 128$ & $207 \pm 14$ & $41 \pm 1.6$ \\
$\quad$ Normal & & & \\
\hline
\end{tabular}

Male Holtzman rats were infected intraperitoneally with 10-20,000 trypanosomes. Normal littermates were not infected. Six animals in each group were exsanguinated at the time $50 \%$ mortality was reached in a control group $(120 \mathrm{~h})$ and approximately $24 \mathrm{~h}$ earlier. All infected rats died within $144 \mathrm{~h}$.

$* \pm 2$ SEM. 


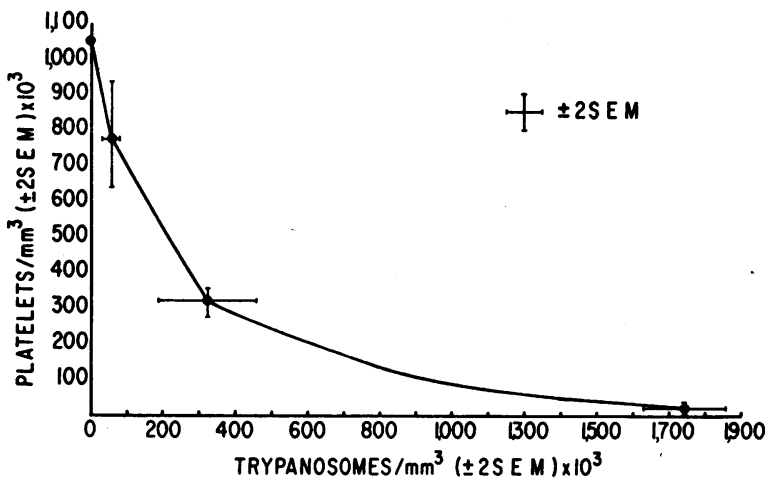

FIGURE 2 Dose response relationship of thrombocytopenia to trypanosomemia. 36 rats were injected on day 0 and at least 6 exsanguinated daily until all were dead. Six uninfected littermates were used as uninfected controls. Trypanosomes and platelets were counted by phase microscopy and the results plotted as platelet counts vs. trypanosome counts. Note the inverse relationship between the numbers of trypanosomes and platelets.

might not show thrombocytopenia or might have a different clinical course with obstruction of small vessels and earlier death. As shown in Table II, splenectomized animals also displayed thrombocytopenia after infection. No platelet aggregates were seen. There was no difference in the clinical course or the time of $50 \%$ or $100 \%$ mortality of infected splenectomized or nonsplenectomized animals.

To rule out the possibility that the thrombocytopenia occurred in vitro by reaction of intact trypanosomes and platelets between the time of exsanguination and the platelet count, six infected rats were treated with suramin $(3 \mathrm{mg} / \mathrm{kg}) 24 \mathrm{~h}$ before the estimated time of death. When they were exsanguinated $24 \mathrm{~h}$ later, the trypanosomemia had cleared, but striking thrombocytopenia persisted with a mean platelet count of $128 \pm 62$ $\times 10^{3}$ platelet $/ \mathrm{mm}^{3}( \pm 2 \mathrm{SEM})$ and was nearly as severe

TABLE II

Effect of Trypanosomiasis on Platelet Counts, Fibrinogens, and HCT of Splenectomized Rats

\begin{tabular}{cccc}
\hline Rats & Platelet count & Fibrinogen & HCT \\
\hline & $\times 10^{3} / \mathrm{mm}^{\mathrm{s}}$ & $\mathrm{mg} / 100 \mathrm{ml}$ & $\%$ \\
Uninfected & $1,200 \pm 68$ & $227 \pm 24$ & $43 \pm 2$ \\
Infected & $204 \pm 56$ & $251 \pm 16$ & $43 \pm 5$ \\
\hline
\end{tabular}

Holtzman male rats (200-250 g) were splenectomized under ether anesthesia. $4 \mathrm{wk}$ later when the platelet counts were approaching normal, 15 rats were infected intraperitoneally with 10,000 trypanosomes. When $50 \%$ of the infected rats had died (5 days), five infected rats and five uninfected, splenectomized rats were exsanguinated and studied. All infected, splenectomized rats died within $144 \mathrm{~h}$. Values are \pm SEM.
TABLE III

In Vitro Effect of Nonmotile Trypanosoma rhodesiense on Rat Platelets, White Cells, and Hct

\begin{tabular}{lccc}
\hline Effect of $0.1 \mathrm{ml}$ of & Platelet count & White cells & HCT \\
\hline & $\times 10^{3} / \mathrm{mm}^{\mathbf{3}}$ & & $\%$ \\
Rat plasma & $998 \pm 19^{*}$ & 15,400 & 37.5 \\
Trypanosomes & $113 \pm 9$ & 14,050 & 37.0 \\
\hline
\end{tabular}

$* \pm 2$ SEM.

TABLE IIIa

Aggregation of Rabbit and Human Platelets by Trypanosoma rhodesiense

\begin{tabular}{lcc}
\hline \multirow{2}{*}{ Effect of $0.1 \mathrm{ml}$ of } & \multicolumn{2}{c}{ Platelet counts \pm 2 SEM } \\
\cline { 2 - 3 } & Rabbit & Human \\
\hline & \multicolumn{2}{c}{$\times 10^{3} / \mathrm{mm}^{3}$} \\
Homologous plasma & $715 \pm 136$ & $269 \pm 33$ \\
Trypanosomes & $65 \pm 13$ & $147 \pm 15$ \\
\hline
\end{tabular}

Trypanosomes were harvested from the top of the buffy coat of infected rats. To $1.0 \mathrm{ml}$ of whole rat blood anticoagulated with EDTA, $0.1 \mathrm{ml}$ of normal rat plasma or concentrated trypanosomes $\left(10^{8}\right)$ was added. The trypanosome suspensions are poor in platelets, white cells, and red cells. The tubes were rocked on an Ames aliquot mixer at room temperature and cell counts and HCTS measured at $30 \mathrm{~min}$. All platelets that could be counted were included in the tally including those in small aggregates of four or five. Most of the platelets in the blood treated with trypanosomes were in aggregates of greater than 10, and aggregates of greater than 50 were not uncommon.

The figures in Table III represent 14 paired determinations for platelets and five paired determinations for white cells and HCT. The figures in Table IIIa represent the effect on the platelets of five individuals studied simultaneously.

as that of six infected littermates bled $24 \mathrm{~h}$ earlier ( 62 $\left.\pm 36 \times 10^{3}\right)$ at the time the treated rats were given suramin. Suramin $(3 \mathrm{mg} / \mathrm{kg})$ did not alter the platelet count of six uninfected rats $\left(1,053 \pm 60 \times 10^{3}\right)$ compared to six untreated littermates $\left(1,042 \pm 40 \times 10^{3}\right)$. This experiment did not rule out an effect of circulating trypanosomal products on platelets.

\section{In vitro platelet aggregation}

Addition of $10^{7}$ to $10^{8}$ trypanosomes to $1 \mathrm{ml}$ of normal rat blood invariably caused platelet aggregation in vitro. Nonmotile trypanosomes stored in the refrigerator for up to 1-2 wk were as effective as fresh, motile trypanosomes. A typical experiment is shown in Table III. After $30 \mathrm{~min}$ most of the platelets were found in aggregates of greater than 10, and aggregates of 50 were not uncommon. As shown in Table III, there was no effect on the HCT or white cell count. Multiple determinations have shown no effect on fibrinogen con- 
centration. FSP were not studied in vitro. The aggregation phenomenon occurred equally in whole blood or $\mathrm{PRP}$ at temperatures of $22^{\circ}-37^{\circ} \mathrm{C}$ and was effective in blood anticoagulated with EDTA, sodium citrate, or heparin. Heparin was not used routinely, however, because spontaneous aggregation occurred in heparinized rat blood.

Aggregation significantly lowered the platelet count by $15 \mathrm{~min}$ and was virtually complete by $30 \mathrm{~min}$. There was no significant change between 30 and $180 \mathrm{~min}$. Continued oscillation at $36 / \mathrm{min}$ or rapid vortex mixing for $30 \mathrm{~s}$ (Vari Whirl, Van Waters and Rogers, Inc., San Francisco, Calif.) did not change the platelet count or the number of aggregates. Vigorous agitation on a Burrell Wrist Action Shaker (Burrell Corp., Pittsburgh, Pa.) at $210 \mathrm{cycles} / \mathrm{min}$ for $5 \mathrm{~min}$ physically sheared some aggregates, increasing both the number of aggregates and the number of platelets. When a 30 min preparation with a platelet count of $28 \times 10^{3} / \mathrm{mm}^{3}$ and $26 \times 10^{3}$ large aggregates $/ \mathrm{mm}^{3}$ was subjected to 5 min agitation on the wrist action shaker, the platelet count increased to $184 \times 10^{3} / \mathrm{mm}^{3}$ and the number of aggregates to $76 \times 10^{3} / \mathrm{mm}^{3}$.

As shown in Table IIIA, trypanosomes also caused aggregation of human and rabbit platelets.

\section{Mechanism of platelet injury}

Because platelet aggregation was not detected at any stage of infection, it was possible that the in vivo thrombocytopenia was not related to in vitro aggregation. For example, the in vitro phenomenon might be ADP-induced aggregation that would not occur in vivo because the smaller circulating concentrations of ADP available in vivo at any given time might be hydrolyzed to inactive metabolic products. It did not seem likely, however, that the aggregation was due to ADP because it occurred in the presence of strong chelating agents such as EDTA, known to interfere with ADP-induced aggregation (12). Other more likely explanations that would relate the in vivo and in vitro phenomena included immunologic injury and platelet damage by a trypanosomal enzyme or toxin. Immunologic injury would most likely be mediated by antigen-antibody complexes with platelet injury by immune adherence, late components of complement, or pharmacologically active substances such as kinins.

Dose-response relationships. An inverse relationship between the numbers of trypanosomes and platelets in both the in vivo and in vitro models first suggested that thrombocytopenia in vivo and aggregation in vitro might be due to a direct effect of the trypanosomes or their products on the platelets. Further exploration of this relationship showed interesting dose-response curves (Figs. 2 and 3 ). The number of trypanosomes neces-

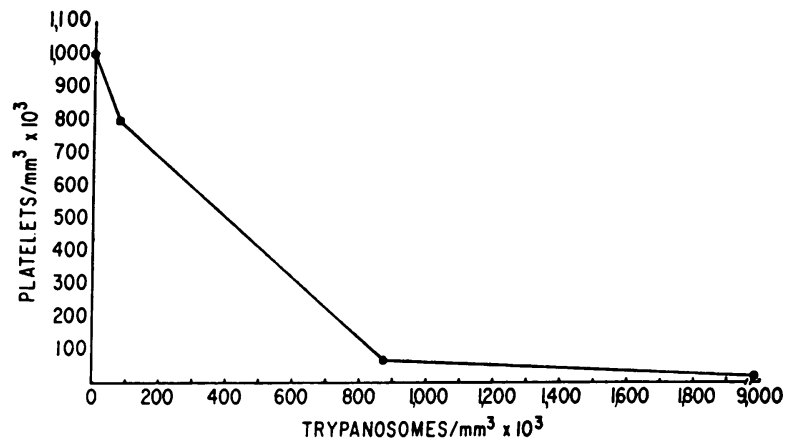

FIGURE 3 Dose response relationship of the aggregation phenomenon to the concentration of trypanosomes. Serial 10 -fold dilutions of concentrated trypanosomes were each added to $1 \mathrm{ml}$ of a pool of normal rat blood and the platelet counts determined after $30 \mathrm{~min}$. The negative control consisted of the same volume $(0.1 \mathrm{ml})$ of normal plasma harvested from the same pool of rat blood. Note that the number of trypanosomes needed to cause aggregation (about $\left.800 \times 10^{3} / \mathrm{mm}^{3}\right)$ is the same as that necessary to cause thrombocytopenia in vivo (Fig. 2).

sary to cause minimal but significant in vivo thrombocytopenia was the same (about $800 \times 10^{3}$ ) as the number necessary to initiate the in vitro aggregation phenomenon. Further, the shapes of the curves and the points of "maximal" effect were similar.

ADP-induced aggregation. The possibility that the in vitro phenomenon was due merely to the addition of exogenous ADP from trypanosomes or hemolyzed red cells was tested and disproved in the following ways: (a) ADP concentrations 6,000 times higher than the usual aggregating dose of $0.3-1.0 \mu \mathrm{g} / \mathrm{ml}$ (13) did not cause aggregation in EDTA-treated whole blood or rat PRP; $(b)$ trypanosome-induced aggregation was not inhibited by $50 \mathrm{mM}$ adenosine, either in EDTA-

TABLE IV

In Vitro Aggregating Effect of Trypanosoma rhodesiense on Rabbit Platelets in a Decomplemented System

\begin{tabular}{cc}
\hline Effect of $0.1 \mathrm{ml}$ of & $\begin{array}{c}\text { C6 deficient } \\
\text { rabbit blood } \\
\text { (collected in } \\
0.2 \text { M EDTA) }\end{array}$ \\
\hline $\begin{array}{c}\text { Trypanosomes harvested } \\
\text { in } 0.2 \text { M EDTA }\end{array}$ & 194 \\
$\begin{array}{c}\text { Normal plasma harvested } \\
\text { in } 0.2 \text { M EDTA }\end{array}$ & 730 \\
\hline
\end{tabular}

Trypanosomes were harvested from rat blood drawn into $0.2 \mathrm{M}$ EDTA and added to C6-deficient rabbit blood also harvested in $0.2 \mathrm{M}$ EDTA. In this system complement mediated immune adherence and $\mathrm{C}$ lysis were both blocked. The platelets were counted at $30 \mathrm{~min}$ as outlined in the text and in Table III. 
TABLE V

Platelet Aggregation by Trypanosomes in the Presence of Kinin Inhibition

\begin{tabular}{|c|c|c|c|c|}
\hline \multicolumn{5}{|c|}{ Trypanosomes pretreated with: } \\
\hline $\begin{array}{c}\text { Saline control } \\
\text { (no trypanosomes) } \\
1,100\end{array}$ & $\begin{array}{c}\text { Untreated } \\
168\end{array}$ & $\begin{array}{c}\text { Trypsin } \\
(50 \mu \mathrm{g} / \mathrm{ml}) \\
132\end{array}$ & $\begin{array}{c}\text { Alpha-chymotrypsin } \\
(50 \mu \mathrm{g} / \mathrm{ml}) \\
136\end{array}$ & $\begin{array}{c}\text { Lima bean } \\
\text { trypsin inhibitor } \\
(125 \mu \mathrm{g} / \mathrm{ml}) \\
162\end{array}$ \\
\hline
\end{tabular}

Platelet count $\times 10^{3} / \mathrm{mm}^{3}$

Platelet counts from pooled blood of five rats. After harvesting, trypanosomes were treated with the indicated concentration of trypsin or alpha-chymotrypsin for $30 \mathrm{~min}$ at $37^{\circ} \mathrm{C}$. Trypsin was inactivated by lima bean trypsin inhibitor before trypanosomes were added to the rat blood. Note that the lima bean trypsin inhibitor also had no effect on aggregation. Platelets were counted at $30 \mathrm{~min}$ as outlined in the text and in Table III.

plasma or heparinized plasma. At this concentration adenosine is a powerful inhibitor of ADP-induced platelet aggregation (13); (c) removal of ADP from trypanosome concentrates by dialysis and Sephadex QAE50 did not alter the aggregating effect. At the same concentration of trypanosomes $\left(8 \times 10^{8} / \mathrm{mm}^{3}\right)$, aggregation resulted in platelet counts of $28 \times 10^{3} / \mathrm{mm}^{3}$ with the usual buffy coat trypanosomes, $34 \times 10^{3}$ with dialyzed trypanosomes, and $26 \times 10^{3}$ with column trypanosomes. The plasma control was $1,100 \times 10^{3} / \mathrm{mm}^{3}$.

Complement inhibition. Because immune complexes in the presence of complement may cause platelet aggregation, we tested this possibility in vitro by the following experiment. The blood of rabbits congenitally deficient in $\mathrm{C} 6$ was drawn into $0.2 \mathrm{M}$ EDTA, a concentration sufficient to inactivate $\mathrm{C} 4$ and $\mathrm{C} 2$. In this system immune lysis requiring all late components of $\mathrm{C}$ and immune adherence requiring the first four components would be completely blocked. As shown in Table IV, platelet aggregation is not mediated by complement.

Proof that the in vivo thrombocytopenia was also independent of the late components of complement at least was obtained by checking for thrombocytopenia in infected C6-deficient rabbits. Two rabbits congenitally

TABLE VI

Effect of Particle-Free Supernate from Disrupted Trypanosomes on Rat Platelets

Platelet counts $\times 10^{3} / \mathrm{mm}^{3} 30 \mathrm{~min}$ after $1.0 \mathrm{ml}$ of rat blood was treated with $0.2 \mathrm{ml}$ of :

\begin{tabular}{ccc} 
of rat blood was treated with $0.2 \mathrm{ml}$ of : \\
\hline $\begin{array}{ccc}\text { Normal } \\
\text { plasma }\end{array}$ & Supernate & $\begin{array}{c}\text { Heapernate } \\
56^{\circ} \mathrm{C} \text { for } \\
30 \mathrm{~min}\end{array}$ \\
\hline 1,038 & 452 & 896 \\
\hline
\end{tabular}

Normal blood anticoagulated with EDTA was obtained from a pool of five rats. Platelets were counted at $30 \mathrm{~min}$ as outlined in the text and in Table III. deficient in $\mathrm{C} 6$ were infected in the usual manner. 3 wk later, when the animals began to show signs of clinical illness, platelet counts were compared to those taken 2 days before infection. The platelet counts before infection were $650 \times 10^{3}$ and $852 \times 10^{3} / \mathrm{mm}^{3}$. The 21 -day post-infection counts were $122 \times 10^{3}$ and $210 \times 10^{3} / \mathrm{mm}^{3}$, respectively.

Kinin inhibition. Kininlike substances, however, might be produced and released directly from trypanosomes, from platelets, or from rat mast cells. The rabbit plasma inactivator of kinin, kininase, is destroyed by EDTA. Such inactivation of trypanosomal kinin might explain the persistence of the in vitro platelet aggregation by trypanosomes in the presence of this chelating agent. Cochran and Wuepper (14) have shown that kinin activity is destroyed by carboxypeptidase- $B$ or alpha-chymotrypsin but not by trypsin. The experiment shown in Table $\mathrm{V}$ exploited this observation. As shown in this and several other experiments, neither alpha-chymotrypsin or trypsin inhibited tryanosome-induced platelet aggregation at the usual concentrations shown in Table V $(50 \mu \mathrm{g} / \mathrm{ml})$ nor in concentrations of $500 \mu \mathrm{g} / \mathrm{ml}$.

Studies implicating a possible trypanosomal enzyme or toxin. Because the thrombocytopenia was not due to immune complexes, $\mathrm{ADP}$, or kinin activity, it might be mediated by a trypanosomal enzyme or toxin. Weitz (15) found an exoantigen of Trypanosoma brucei in the serum of infected animals and in washings of harvested trypanosomes. This antigen was necessary for infectivity, because infectivity for mice was lost by washing trypanosomes and restored by suspending the organisms in their washings or trypanosome-free infected serum. Platelet aggregation, on the other hand, was not affected by washing our strain of Trypanosoma rhodesiense. Harvested trypanosomes washed three times in 10 vol of $0.15 \mathrm{M}$ saline caused platelet aggregation equal to fresh organisms. Concentrated, infected plasma and the concentrated washings failed to cause aggregation. Since the aggregating factor appeared to be bound 
to the trypanosome, we next tried to isolate the active factor by disruption of the organisms. This manipulation was successful only after very short periods of sonication. When $0.2 \mathrm{ml}$ of the trypanosome-free supernate of the disrupted trypanosomes was added to 1.0 $\mathrm{ml}$ of normal rat blood, aggregation always occurred. A typical experiment is shown in Table VI. Like the whole trypanosome, this aggregating factor is effective in EDTA-treated blood and is resistant to kinin inhibitors. It is labile, however, to heating at $56^{\circ} \mathrm{C}$ for $30 \mathrm{~min}$ (Table VI). This heat lability suggests that the aggregating substance may be a protein toxin or enzyme. Its effectiveness in the presence of EDTA and the previous studies with the whole trypanosome seem to rule out the participation of complement.

The aggregating substance was not removed from the particle-free supernate by dialysis. Column-purified trypanosomes sonicated and made particle-free by centrifugation were divided into two portions. After overnight dialysis of one portion against PSG buffer, equal volumes of the two portions caused equal platelet aggregation in citrated rat PRP. After $30 \mathrm{~min}$ the platelet counts were; normal rat plasma control $1,450 \times 10^{3}$, untreated supernate $346 \times 10^{3}$, and dialyzed supernate $378 \times 10^{3} / \mathrm{mm}^{3}$.

$E M$. Scanning and transmission electron micrographs were made of aggregates induced by whole trypanosomes and trypanosome-free supernate. Fig. 4 shows the typical appearance on scanning EM of an aggregate induced by the trypanosome-free supernate. Fig. 5 compares normal platelets and aggregated platelets by transmission EM. These aggregates show typical changes of aggregation, including degranulation, centralization of granules, presence of micelles, and reduction in cytoplasmic density.

\section{DISCUSSION}

Severe thrombocytopenia occurs in rats and rabbits infected with this human strain of Trypanosoma rhodesiense. Since it occurs without consumption of fibrinogen or release of FSP (Table I and Fig. 1), it is clear that the thrombocytopenia is not secondary to DIC. Instead a direct injury of platelets by trypanosomes appears responsible. Direct injury was first suggested by the inverse relationship of parasitemia to thrombocytopenia (Fig. 2). The degree of platelet aggregation caused by the addition of trypanosomes in vitro to normal rat, rabbit, or human blood (Table III and IIIA) was directly related to the number of trypanosomes (Fig. 3) and stimulated a search for a trypanosomal factor capable of causing direct platelet damage. After it was shown that the in vitro aggregation was not caused by ADP derived from trypanosomes or red cells, this system became a simple assay for

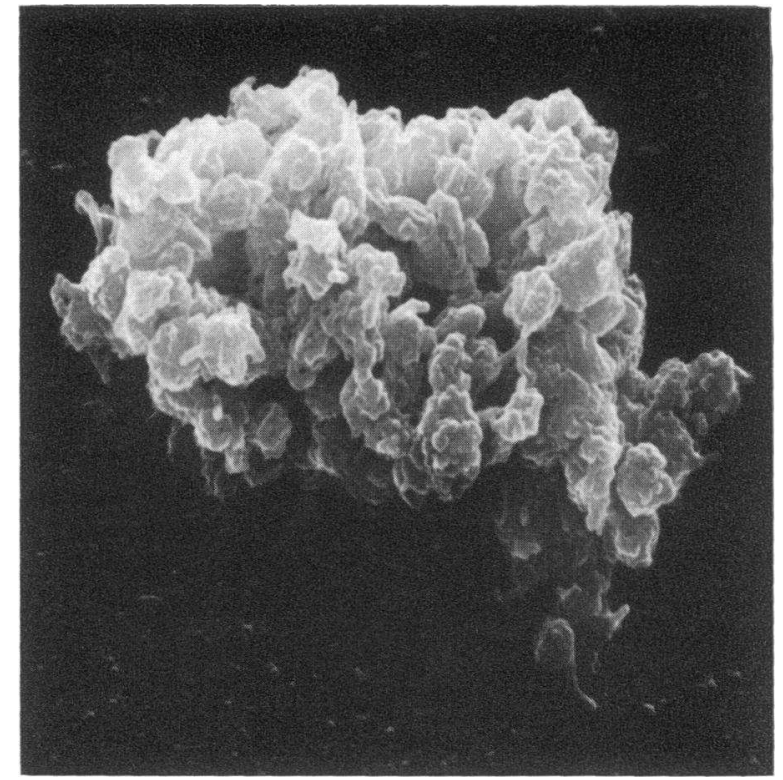

FIGURE 4 Platelet aggregate (at $90 \mathrm{~min}$ ) induced by trypanosome-free supernate $\times 2,900$ (scanning $\mathrm{EM}$ ).

studying the mechanism of platelet injury. A series of experiments with this system, supplemented by the in vivo model, has ruled out immunologic injury as the cause of platelet damage and shown that the aggregation is caused by a substance found in heat-labile supernates of disrupted trypanosomes.

It was necessary to study immunologic phenomena carefully because a series of reports have implicated the participation of antigen-antibody complexes in the pathogenesis of the lesions of trypanosomiasis. First, in experimental rabbit trypanosomiasis, each wave of parasitemia is caused by trypanosomes with antigens different from those of the predecessors, against which antibody has already been produced (16). Second, Goodwin and Hook (17) have shown that the major pathologic changes in rabbits infected with Trypanosoma brucei are vascular lesions, thrombus formation, and increased vascular permeability. Finally, kinin concentrations are increased in experimental infections of laboratory animals (18) and volunteers (19). Thus it has been postulated that the vascular pathology is related to the release of kinins by antigen-antibody complexes. Our studies suggest an alternative mechanism of vascular damage by showing that trypanosome mediated platelet injury is independent of immune complexes as mediated by immune adherence (Table IV), complement-mediated lysis (Table IV), and kinins (Table V). Independence from late components of complement was also shown by demonstrating that infected C6-deficient rabbits also developed thrombocytopenia. It is not known whether the trypanosomes in Goodwin and 

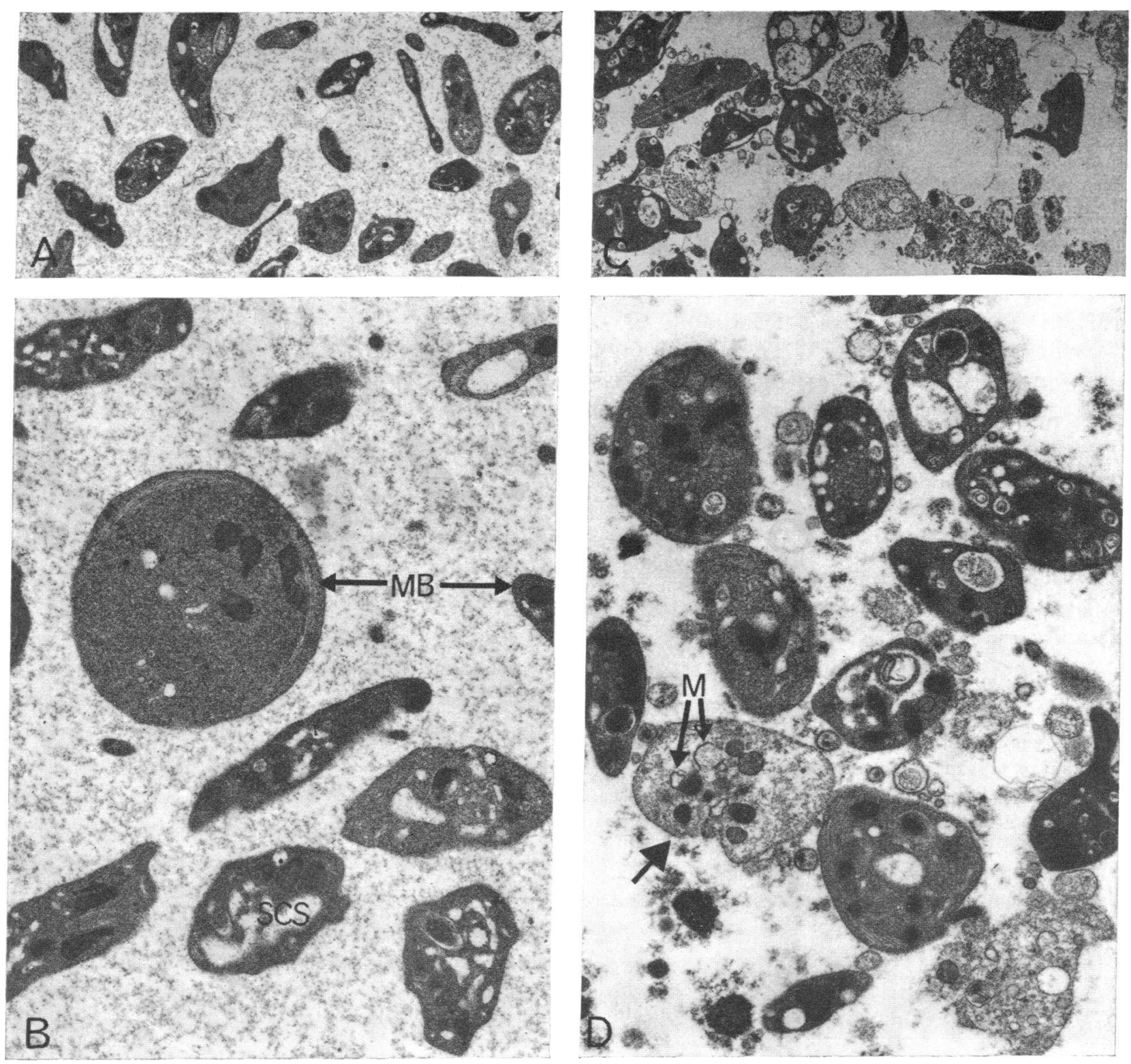

FIGURE 5 Comparison of normal rat platelets and platelet aggregates induced by trypanosome-free supernate. Aggregates were induced by adding $0.2 \mathrm{ml}$ of trypanosome-free supernate to $1 \mathrm{ml}$ of rat PRP. Controls were PRP to which $0.2 \mathrm{ml}$ of platelet-poor plasma from the same pool was added. Samples were obtained after $90 \mathrm{~min}$ as noted in the text.

A. Control platelets $\times 5,800$.

B. Same as $A \times 12,500$. Note marginal band of microtubules $(\mathrm{MB})$ in longitudinal and cross-sections and surface-connected membrane system (SCS).

C. Platelet aggregate $\times 5,800$.

D. Same as $C \times 12,500$. Note degranulation and reduction of cytoplasmic density in several platelets, with some micelles (M) and free granules present and centralization of granules (large arrow).

Hook's rabbits (17) or Boreham's volunteers (19) caused thrombocytopenia, because platelets were not studied. Deposition of platelets in the vascular lesions described by Goodwin and Hook could also contribute to thrombocytopenia. Gross and microscopic examination of the kidneys, livers, spleens, lungs, hearts, and 
brains of rats infected with our strain of $T$ rhodesiense has not demonstrated such vascular lesions or fibrin deposition. Splenomegaly is the only finding.

As shown in Table VI, remarkable platelet aggregation is produced by trypanosome-free extracts of disrupted organisms. The aggregating substance is so labile that it is inactivated by sonication lasting as little as $20 \mathrm{~s}$ beyond the initial 20 -s period. The fact that it is heat-labile but not blocked by inhibitors of complement strongly suggests that it is a protein toxin or enzyme. The identity of this substance has not been determined. Among the agents known to cause platelet aggregation, ADP, antigen-antibody complexes, and FSP have been ruled out. Serotonin and epinephrine are unlikely because of the marked lability of the phenomenon to heat and sonication. Long-chained saturated fatty acids seem to be eliminated because the aggregating substance was not removed from supernates by extraction with petroleum ether. ${ }^{2}$ The trypanosomes may contain a protein with thrombin, fibrinogen, or collagen activity or a trypanosomal protein unrelated to other aggregating substances. Larger quantities of the thrombocytopenic factor are being accumulated for studies in vivo with the aggregating substance and in vitro with protein and enzyme inhibitors and isolation of the aggregating substance.

Thrombocytopenia has not been reported previously in experimental trypanosomiasis and indeed in only two cases of naturally acquired human trypanosomiasis $(1,20)$. Both patients were Americans who visited endemic areas of Africa. It is important to know whether thrombocytopenia is a uniform accompaniment of Rhodesian trypanosomiasis, because the usual cause of death in this fulminating disease is unknown. Our data suggest that thrombocytopenia may be an important manifestation of this illness.

The platelet injury described here is independent of hemolysis, DIC, or immunologic injury related to immune adherence, complement-mediated lysis, or release of kinins. Aggregation of platelets in vitro by heatlabile supernates of disrupted trypanosomes is not blocked by inhibitors of complement or ADP. If the platelet effect is due to a protein toxin or enzyme, this will be the first infectious agent shown to have a direct toxic effect on the platelet without DIC. Release of procoagulants from injured platelets might then result in intravascular clotting in some animal species and account for the DIC observed in the patient treated in this hospital.

Note added in proof. After this manuscript was completed, our observation of thrombocytopenia in human trypanosomiasis was confirmed in rhesus monkeys by Sadun, Johnson, Nagle, and Duxbury. 1973. Am. J. Trop. Med. Hyg. 22: 323.

${ }^{2}$ Unpublished observation.

\section{REFERENCES}

1. Barrett-Connor, E., R. J. Ugoretz, and A. I. Braude. 1973. Disseminated intravascular coagulation in trypanosomiasis. Arch. Intern. Med. 131: 574.

2. Dennis, L. H., J. W. Eichelberger, M. M. Inman, and M. E. Conrad. 1967. Depletion of coagulation factors in drug-resistant Plasmodium falciparum malaria. Blood J. Hematol. 29 : 713.

3. Borochovitz, D., A. L. Crosley, and J. Metz. 1970. Disseminated intravascular coagulation with fatal haemorrhage in cerebral malaria. Br. Med. J. 2: 710 .

4. Hill, G. J., II, V. Knight, and G. M. Jeffrey. 1964. Thrombocytopenia in vivax malaria. Lancet. 1: 240.

5. Neva, F. A., J. N. Sheagren, N. R. Shulman, and C. J. Canfield. 1970. Malaria: host-defense mechanisms and complications. Ann. Intern. Med. 73: 295.

6. Skudowitz, R. B., J. Katz, A. Lurie, J. Levin, and J. Metz. 1973. Mechanisms of thrombocytopenia in malignant tertian malaria. Br. Med. J. 2: 515.

7. Hoffman, T. A., and E. A. Edwards. 1972. Groupspecific polysaccharide antigen and humoral antibody response in disease due to Neisseria meningitidis. $J$. Infect. Dis. 126: 636.

8. Ratnoff, O. D., and C. Menzie. 1951. New method for determination of fibrinogen in small samples of plasma. J. Lab. Clin. Med. $37: 316$.

9. Leavelle, D. E., B. F. Mertens, E. J. W. Bowie, and C. A. Owen, Jr. 1971. Staphylococcal clumping on microliter plates. A rapid simple method for measuring fibrinogen split products. Am. J. Clin. Pathol. 55: 452.

10. Braude, A. I., H. Douglas, and C. E. Davis. 1973. Treatment and prevention of intravascular coagulation with antiserum to endotoxin. J. Infect. Dis. 128: S157.

11. Lanham, S. M. 1968. Separation of trypanosomes from the blood of infected rats and mice by anion-exchangers. Nature (Lond.). 218: 1273.

12. Born, G. V. R. 1967. Mechanism of platelet aggregation and its inhibition by adenosine derivatives. Fed. Proc. 26: 115 .

13. Born, G. V. R., A. J. Honour, and J. R. A. Mitchell. 1964. Inhibition by adenosine and by 2-chloroadenosine of the formation and embolization of platelet thrombi. Nature (Lond.). $202: 761$.

14. Cochrane, C. J., and K. A. Wuepper. 1971. The kininforming system in plasma. Excerpta Med. Int. Cong. Ser. 229: 137.

15. Weitz, B. 1960. The properties of some antigens of Trypanosoma brucei. J. Gen. Microbiol. 23: 589.

16. Gray, A. R. 1962. The influence of antibody on serological variation in Trypanosoma brucei. Ann. Trop. Med. Parasitol. 56: 4.

17. Goodwin, L. G., and S. V. M. Hook. 1968. Vascular lesions in rabbits infected with Trypanosoma (Trypanozoon) brucei. Br. J. Pharmacol. Chemother. 32: 505.

18. Richards, W. H. G. 1965 . Pharmacologically active substances in the blood, tissues, and urine of mice infected with Trypanosoma brucei. Br. J. Pharmacol. Chemother. $24: 124$.

19. Boreham, P. F. 1970. Kinin release and the immune reaction in human trypanosomiasis caused by Trypanosoma rhodesiense. Trans. R. Soc. Trop. Med. Hyg. 64: 394.

20. Ottoman, P., J. Zumwalt, J. Chin, and R. Roberto. 1970. African trypanosomiasis-California. Morbid. Mortal.19: 233. 